\title{
Comparison of Towed Conductivity Sensor Performance
}

\author{
J. P. Dugan and B. W. Stalcup . \\ Arete Associates, Arlington, Virginia \\ (Manuscript received 12 February 1987, in final form 12 August 1987)
}

\section{ABSTRACT}

\begin{abstract}
Electrical conductivity sensors are often used to obtain measurements of small-scale fluctuations, or microstructure, in the ocean. In applications on towed instrument packages, they provide the only way to estimate temperature fluctuations on scales down to about $1 \mathrm{~cm}$. A number of designs for the sensors have emerged in the last few years which address the major requirements of the instruments, namely, high spatial resolution and freedom from fouling. Five specific designs are compared on the basis of these design objectives, with the intent of identifying those which most successfully reach the goals. A dual-needle cell is found to have very high spatial resolution, and a surface-mounted planar sensor is found to be most immune to fouling. Other sensors, such as the conductivity cell used in the Neil Brown Instrument Systems CTD, are found to be less optimum in this application.
\end{abstract}

\section{Introduction}

Fundamental problems in physical oceanography involve the transport and mixing of heat, mass, momentum, and chemical pollutants. For instance, a complete understanding of the physical processes underlying mixing is necessary if the oceanographer is to understand fully the ocean's basic density structure (Garrett, 1979) and the transport of anthropogenic pollutants through this structure (Pritchard, 1960). This understanding has been limited by the paucity of observational data on mixing in the ocean interior; specifically on the location, duration, and intensity of significant mixing events. These observational data have not been available until recently because of difficulties in the development of appropriate systems. These systems must obtain reasonably large samples of data (Gibson, 1981) from a number of interesting locations (Gregg and Briscoe, 1979) so that the appropriate studies can be performed. In order to do this, these systems are generally towed (Nasmyth, 1980), and they often employ electrical conductivity sensors in order to obtain good spatial resolution at substantial forward speeds (Washburn and Gibson, 1982).

A critical requirement for estimating the intensity of mixing of scalar quantities is the ability to measure the variance in the conductivity (or temperature) gradient, which requires spatial resolution approaching 1 $\mathrm{cm}$. For typical tow speeds of $5 \mathrm{kt}$ or faster, this requires a sensor frequency response of $250 \mathrm{~Hz}$ or better. Conductivity sensors are good candidates for this application because they typically can be built to obtain

Corresponding author address: Dr. John P. Dugan, Arete Association, P.O. Box 16287, Arlington, VA 22215. high-frequency response while achieving very good ruggedness, dependability, and ease of operation. Other choices of sensors have included thermistors and thin film resistance thermometers, but the former is limited to frequency response of less than $10 \mathrm{~Hz}$ (Fabula, 1968; Lueck et al., 1977), and the latter has been difficult to maintain and operate. Gregg and Pederson (1980) and Gibson and Deaton (1980) further discuss alternative measurement techniques.

Since the measurement of electrical conductivity is usually made by a resistance cell, the inherent frequency response of the sensor is very high. For an ideal sensor, the spatial response would be determined only by the cell geometry (see Gregg et al., 1982). The effective "length" of the cell is a direct function of the geometry imposed on the current field, and the resulting effective frequency response is given simply by the ratio of the speed through the water to this cell "length". Real sensors, however, partially retard the flow through the action of fluid boundary layers. This effect is expected to degrade the effective spatial resolution of some sensor geometries more than others (cf. Topham and Perkin, 1984).

A second requirement for these sensors is that they have sufficient dynamic range so that they stay on scale and yet are able to resolve the relatively low-signal level of microstructure above system noise. Since this microstructure signal can be in the range of several tenths of microsiemans $/ \mathrm{m}$ ( 1 sieman $/ \mathrm{m}=10 \mathrm{mmho} / \mathrm{cm}$ ) while the mean signal level is several siemans $/ m$, the required dynamic range is wider than six decades of amplitude. Fortunately, the microstructure signal occurs at high frequencies while the mean signal level is a low frequency occurrence. This permits the signal to be split into high- and low-frequency components or 
to be electronically preemphasized so that it can be recorded conveniently either in digital or analog form. In either case, the driver and amplifier electronics must be of low noise design to accomplish the objective.

There is a design trade-off among these requirements in that the best cell geometry for one objective is not conducive to achieving another one. For instance, the spatial resolution can be improved in principle simply by building a very small cell. Unfortunately, this results in a very short current path, and the nominal cell impedance in seawater is quite small. Ultimately, this leads to an elevated noise level and/or calibration drift which limits the dynamic range, so some compromise must clearly be found.

An additional constraint on the cell geometry is that it be relatively immune to biological fouling. The specific type of geometry to avoid is the one which can accumulate matter present in seawater; biota, detritus, etc., in the water will pass through the cell and be sensed by the electrical field causing "spikes" in the data (Okawa and Dugan, 1984). This practically cannot be avoided; the real issue is to avoid this matter impacting the cell and getting caught on any part in the sensing volume. When this occurs, the quality of the data is degraded in two ways. The impacting material biases the cell constant so that the calibration is upset for as long as the matter remains on the cell. Also, the matter typically vibrates rapidly in the flow field so that a considerable amount of high-frequency noise results. The noise level frequently is higher than conductivity signal levels encountered in the ocean, so the sensor is rendered useless. Meagher et al. (1982), Okawa and Dugan (1984), and Farruggia and Fraser (1984) discuss open cells which are optimized to reduce the incidence of this type of fouling.

In this paper, the degree of meeting the above design goals is examined in the case of four specific conductivity cells with which we have worked. Data are reviewed for frequency response, noise level, immunity to fouling, and calibration stability. These performance aspects are also reviewed for an additional sensor which has sufficient information available in the literature. The intent of this paper is to provide potential users with information concerning these areas of sensor performance to assist in their selection of the appropriate sensor for their specific application. Hence, detailed information concerning the operation, circuitry, and construction of these sensors are not provided. Those readers desiring more detailed information about a specific conductivity sensor are directed to either the manufacturers or the references cited in this paper for that sensor.

\section{Conductivity cells}

Conductivity in the ocean is inferred from the resistance measured across some seawater path whose length is dependent upon the specific construction of the conductivity cell. To accomplish this, an alternating electric field is set up in the seawater by applying alternating voltages, switched at a relatively high frequency of several kilohertz or so, to a set of platinum electrodes, referred to as the current source electrodes. The resulting voltage drop across the seawater path is measured by another set of platinum electrodes, referred to as the sensing electrodes. The conductivity signal is then calculated from the resultant impedance. Due to the limited dynamic range of analog to digital converters, the high frequency part of this signal typically is preemphasized to provide adequate resolution of the conductivity signal down to small wavelength scales, and hence high frequency.

Of the four cells examined in detail here, three of them were tested while the authors were associated with the Naval Research Laboratory. Figure 1 is a photograph of these three cells. The fourth one has been tested by the Applied Physics Laboratory of The Johns Hopkins University. A photograph of this cell is not readily available but can be seen in Farruggia and Fraser (1984). For this case, test data have been made available to us. All cells were tested in a towed configuration in the seasonal thermocline of the Sargasso Sea where conductivity signals are dominated by temperature fluctuations.

The sensor which has received the most study is the square cell produced by Neil Brown Instrument Systems (NBIS). It is employed in the Mark III CTD which is widely used in the oceanographic community. The standard $3 \mathrm{~cm}$ long, square cross-sectional cell, as well as a shorter $1.5 \mathrm{~cm}$ square cell, and the older, smaller $\mathrm{D}$ cell of the same manufacturer have been studied for their spatial response by Gregg et al. (1982). The 1.5 $\mathrm{cm}$ cell, shown in the lower left corner of Fig. 1, es-

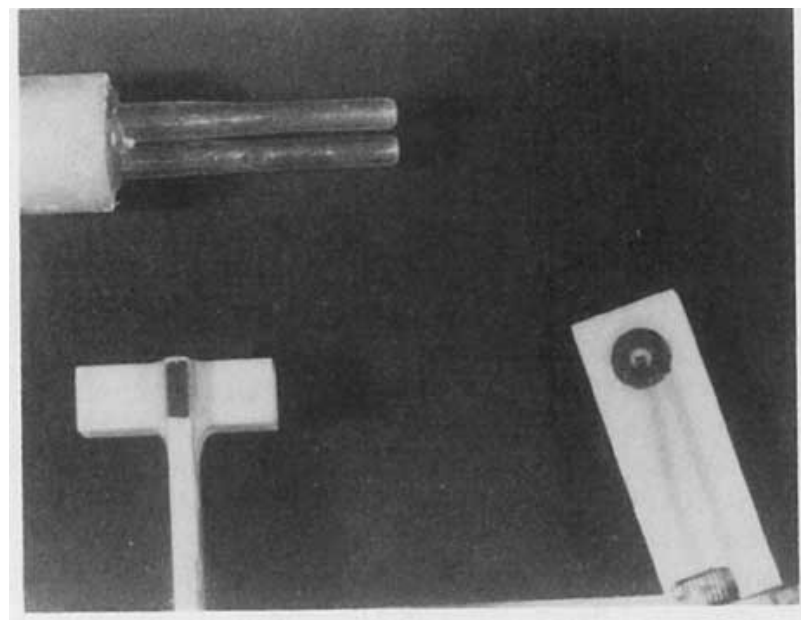

FIG. 1. Photograph of three conductivity cells: (upper left) the APL/ UW needle, (lower left) the $1.5 \mathrm{~cm}$ NBIS square, and (lower right) the NBIS planar. The direction of flow in this photograph is from right to left, and, for comparing length scales, the Neil Brown sensor is $1.5 \mathrm{~cm}$ long. 
pecially has been employed in towed configurations because it is readily available, and it has the advantage of better spatial response over the standard $3 \mathrm{~cm}$ cell. The response of this cell is down by $3 \mathrm{~dB}$ at a scale of about $7 \mathrm{~cm}$ and exhibits a rather complicated functional form on shorter scales. It has a sequence of nulls in the response, with the first one being near $1.7 \mathrm{~cm}$. The subsequent peak in the response near $0.8 \mathrm{~cm}$ is less than an order of magnitude down in power from the DC level. This cell was designed for use in a CTD and it therefore has very good calibration stability. Unfortunately, as will be discussed in detail later, this cell has a tendency to foul easily. This sensor has four electrodes mounted coincident with the supporting strut, shown in Fig. 1, two inside the square cell and two outside. The pair of electrodes on one side of the cell, one inside and one outside, serves as the current source while the remaining two serve as the sensing electrodes.

The second cell is a planar "bull's eye". design which is shown in the lower right corner of Fig. 1. This cell, which was built by NBIS under special order, uses a four-electrode design with electrodes placed on opposite sides of a flat ceramic substrate. The intent of this design was to retain a reasonably small effective cell size, to maintain the four electrode capability, and to improve the immunity to fouling. Each side of the ceramic substrate contains a current source, the circular electrode, which is surrounded by a voltage sensing electrode, the annular ring. The leading edge is glassed over to reduce the chance of organisms catching on the cell.

The third cell is a two electrode design which was constructed by APL/UW (Meagher et al., 1982) and is shown in the upper left corner of Fig. $1 .{ }^{1}$ The electrodes are at the tips of two parallel needles which are pointed directly into the flow. The effective cell size is a direct function of the distance between the tips and, for the cells tested here, they were about $3 \mathrm{~mm}$ apart. This design is simple and its construction requires no special materials or skills, but it necessarily suffers larger calibration drift since the same electrodes are used to drive the current and sense the voltage drop across the cell.

The fourth cell is a four-electrode design which therefore retains good calibration stability but which trades off frequency response to attain high immunity to fouling. It was designed and constructed by APL/ JHU, and details are available in Farruggia and Fraser (1984). It essentially is a variant of the NBIS planar cell discussed above in which all electrodes are mounted flush on one side of a fairing. For this cell, the middle annular ring electrode is the current source while outer rings provide the return path. A middle disc electrode together with two outer discs, which are shorted together, are the voltage sensing electrodes. The absence of any appendages provides the immunity to fouling and also provides substantial ruggedness.

\footnotetext{
'A variant of this design currently is available from Sea-Bird Electronics.
}

An additional cell which has been reasonably well documented in the literature, but which has not been tested by the authors, is a miniature four-electrode design (Washburn and Gibson, 1982). This cell contains four parallel electrodes mounted on one side of a ceramic substrate. Each electrode is a gold rectangular strip $0.3 \mathrm{~mm}$ wide by $2.4 \mathrm{~mm}$ long. For this cell, the two inner electrodes serve as the ac current source while the two outer electrodes sense the resulting voltage fluctuations. Because of the very close spacing of the electrodes, this design is expected to have very good spatial response but perhaps more limited capability in the areas of calibration stability and perhaps noise level. Its vulnerability to fouling is entirely undocumented.

Several other sensor designs have been used at sea, but not enough details of their performance are available to allow direct comparisons beyond the qualitative comments made in this paragraph. For example, Magnell (1976) deployed a towed microstructure conductivity sensor which exhibited very good spatial resolution to about $1 \mathrm{~cm}$, but it had a high noise level and poor calibration stability. Washburn and Deaton (1986) used a four-electrode conductivity sensor in a towyo platform. This sensor had a spatial resolution of $\sim 1$ $\mathrm{cm}$. Its calibration stability and immunity to fouling are unreported. Also, a very small $(\sim 1 \mathrm{~mm})$ four-electrode cell has been designed, constructed, and used in a laboratory water tunnel by Head (1983). The noise level, frequency response, and calibration stability in the laboratory have been well documented, but its immunity to fouling and its ruggedness in the field apparently are untested or, at least, unreported. In addition, a number of investigators have used very small, single conductor cells and a large ground plane in laboratory tanks because of their good spatial resolution (cf. Gibson and Schwarz, 1963). However, these sensors suffer large calibration drifts and they are not used at sea.

\section{Comparison of cell performance}

In the following subsections, the various performance parameters are appraised for each of the sensors. They are compared directly in Table 1 . The conductivity units in this paper are siemans/meter $\left(\mathrm{S} \mathrm{m}^{-1}\right)$.

\section{a. Frequency response}

The frequency response of the five cells discussed previously is compared in Fig. 2. The curves are estimates of the response obtained in a number of different ways. The responses of the two NBIS cells, the APL/ UW cell, and the APL/JHU cell are estimated by considering their response to particulates which have transited the cell, as discussed by Okawa and Dugan (1984). In each case, an average of five or six large events is used. (During these tests, the two speeds for the NBIS planar and APL/JHU needle cell were $2.6 \mathrm{~m} \mathrm{~s}^{-1}$ while 
TABLE 1. Comparison of conductivity sensor performance.

\begin{tabular}{lcccc}
\hline \hline Cell type & $\begin{array}{c}\text { Resolution } \\
(\mathrm{cm})\end{array}$ & $\begin{array}{c}\text { Noise level } \\
\left(\times 10^{-10} \mathrm{~S} \mathrm{~m}^{-1} / \mathrm{cm}\right)^{2}\end{array}$ & $\begin{array}{c}\text { Fouling } \\
\text { immunity }\end{array}$ & $\begin{array}{c}\text { Calibration } \\
\text { stability }\end{array}$ \\
\hline APL/UW needle & 1 & $1.5(0.7-3.0)$ & Very good & Fair \\
NBIS planar & 3 & $3.5(3.0-5.0)$ & Poor & Good \\
NBIS square & 7 & $3.6(1.2-8.0)$ & Poor & Excellent \\
APL/JHU planar & 8 & $0.8(0.02-1.5)$ & Excellent & Good \\
UCSD & 0.8 & $2 \times 10^{5}$ & Unknown & Fair \\
\hline
\end{tabular}

* Noise levels in this table are almost certainly due to the sensors' electronic circuitry. The bandwidths over which these noise levels were calculated are comparable for the various instruments. The upper-frequency limits range from 150 to $190 \mathrm{~Hz}$.

the two speeds for the NBIS square and the APL/JHU cells were $3.0 \mathrm{~m} \mathrm{~s}^{-1}$.) The response of the Washburn and Gibson (1982) sensor (hereafter referred to as the UCSD sensor) is estimated on the basis of their model for frequency response and must be considered as ideal rather than as actually measured. The frequency response numbers in Table 1 are the $-3 \mathrm{~dB}$ points of the response functions in Fig. 2.

The response of the $1.5 \mathrm{~cm}$ square NBIS cell is very close to that measured by Gregg et al. (1982) in a specially constructed dynamic response test facility, while that of the APL/UW cell is slightly better than actually observed in the test facility. The additional peaks in the response curve for the NBIS square cell are due to effects associated with the current path which provides a boxcar average of the enclosed fluid in this cell (Gregg et al., 1982). It is clear from these results that the physical size and shape of the cell translate directly into an effective frequency response. The miniature UCSD cell has the best response while the APL/JHU cell has the poorest.

As mentioned above, the method of using particulate spike data to estimate the cell frequency response has been used by Okawa and Dugan (1984) and has been utilized in this analysis for all four of the sensors which are analyzed in detail. This method was chosen because spikes caused by particulate matter streaming through

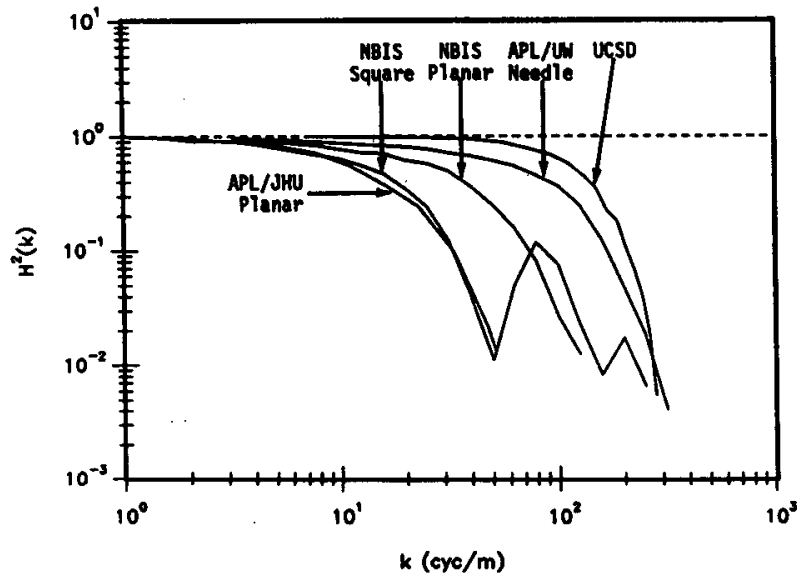

FIG. 2. Frequency response of the conductivity cells. the cell occur in all data with which we have worked, so the method could be applied uniformly to all sensors irrespective of the details of when or where they were deployed. In each case, the frequency spectra of these spikes are white for all frequencies; using this data, the roll-off due to the cell response can be determined easily. An alternative method of determining the response is to observe the spectrum of active microstructure patches in the data. The microstructure typically is expected to involve turbulent flow which should have a spectral slope of $-5 / 3$ (Tennekes and Lumley, 1974). The preemphasis in the processing electronics should increase the spectral slope by a factor of 2 ; thus, the frequency spectrum of turbulence data is expected to exhibit a slope of $1 / 3$. A comparison of spectra of the two types of data is shown in Fig. 3 for the case of the APL/JHU sensor. The upper curve exhibits a slope, which at least is consistent with the $1 / 3$ slope expected for turbulence out to a frequency of $20 \mathrm{~Hz}$, where it

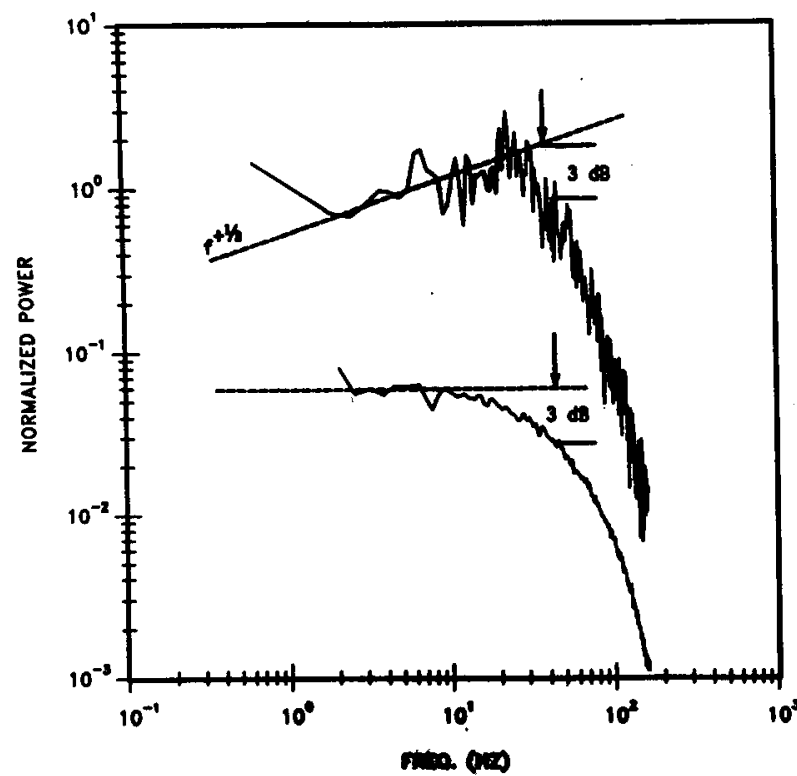

FIG. 3. Comparison of the response of the APL/JHU planar conductivity cell to both particulate matter (lower curve) and microstructure patches (upper curve). The lower curve is offset down 1 decade to avoid overlap. 
rolls off, due to what one presumes is the cell response. The $-3 \mathrm{~dB}$ point of this roll-off occurs near $35 \mathrm{~Hz}$. Concomitantly, the response due to the spikes exhibited in the lower curve is white, and it rolls off with a -3 $\mathrm{dB}$ point near $40 \mathrm{~Hz}$. Within statistical errors, these estimates practically are the same and, for the nominal tow speed of $3.0 \mathrm{~m} \mathrm{~s}^{-1}$, they lead to the estimated spatial response of $8 \mathrm{~cm}$ listed in Table 1. This method of using the response to microstructure patches was not used for all four sensors because the patches are relatively infrequent, they exhibit very large differences in energy level, and there is some chance that the expected $1 / 3$ spectral slope could roll off prematurely for the higher frequencies because of dissipation processes in the ocean rather than sensor response limitations (cf. Caldwell et al., 1980). This latter effect probably explains the faster roll-off of the turbulence spectrum than the spike spectrum at the higher frequencies $(\sim 20 \mathrm{~Hz})$.

\section{b. Noise level}

The noise level measured in situ is the result of a number of individual potential noise sources. The dominant noise typically is due to elements in the detection and amplification circuitry (Lawson, private communication). In this case, the specifics of the particular cell are not important. However, it is possible that noise due to fluctuations in the electrode surface impedance can be a contributing noise source in the limit as the cell size vanishes. Since the electrical conductivity is determined by a resistance measurement across a seawater current path, it is given by an expression of the form

$$
C_{m}=\left(\frac{L}{A \sigma_{w}}+R_{s}\right)^{-1}
$$

where $C_{m}$ is the measured conductance (reciprocal of resistance), $R_{s}$ the surface impedance of the electrodes, $\sigma_{w}$ the specific conductivity of the water, $L$ the effective distance between the electrodes, and $A$ the area of the electrodes. The value of $R_{s}$ depends upon the electrode material and the surface geometry, while the value of the first term depends upon the geometry of the current field. In the search for high resolution, this term becomes smaller as the effective distance between electrodes is made smaller. As this gets small in comparison with $R_{s}$, more amplification must be applied in order to retain sensitivity, and the noise in $R_{s}$ becomes obtrusive. Nevertheless, the dominant noise in all the conductivity sensors reviewed in this paper is believed to be due to the electronic circuitry and not directly to the cell size.

An additional source of noise is discussed separately in subsection $3 \mathrm{c}$ as fouling, but it is mentioned here because of the likelihood of confusion. A low, but constant, level of fouling often is present on the cell, and this is exhibited as (and often is detected by) a higher than normal noise level. Thus, in this comparison, we were careful to review a considerable amount of data from more than one sensor in all cases but that of Washburn and Gibson (1982).

The noise figures in Table 1 for all conductivity sensors, excluding the UCSD sensor, were determined by selection of the minimum values of variances computed over 5-m intervals for each sensor. Each of these values was corrected for the differences in the preemphasis circuitry using the method described in Marmorino et al. (1986) and also for the different tow speeds of 2.6 and $3.0 \mathrm{~m} \mathrm{~s}^{-1}$. The noise figure for the UCSD sensor was obtained from the integral of the noise spectrum reported in Washburn and Gibson (1984) and converting this value into comparable units. All of the noise levels in this table were calculated over similar bandwidths with the upper frequency limit ranging from 150 to $190 \mathrm{~Hz}$.

The two NBIS cells used very similar electronics and both exhibited noise near $3 \times 10^{-10}\left(\mathrm{~S} \mathrm{~m}^{-1} / \mathrm{cm}\right)^{2}$. The APL/UW needle probe is about a factor of 2 better, and the APL/JHU sensor was another factor of 2 better. This latter sensor was the most recently developed and its electronics inevitably were quieter. In each case above, the numbers in brackets in Table 1 give the range of values actually observed in several sensors in one or more deployments. The UCSD sensor exhibited an in situ noise level near $10^{-5}\left(\mathrm{~S} \mathrm{~m}^{-1} / \mathrm{cm}\right)^{2}$ which is much worse than those quoted above. In fact, that sensor had to be deployed at a depth near the maximum temperature gradient in the thermocline to detect microstructure signal levels significantly above the noise. The problem very likely was in the electronics and not due to the cell.

\section{c. Immunity to fouling}

All towed conductivity sensors must be designed so that they do not dredge up biota and detritus. In several days of tows in the Sargasso Sea, a NBIS square cell performed poorly in this aspect. Out of about $100 \mathrm{~h}$ of towing, approximately $35 \%$ of the data exhibited very high noise level due to attached material, and the data were classified as useless. An additional $30 \%$ of the data were classified as being of questionable usefulness because of an elevated noise level due to a lower degree of fouling. In an independent deployment, the unusable portion of data was $24 \%$, with the portion of questionable use being $38 \%$. In the case of elevated noise level, some higher-energy microstructure events could be detected in the data, but many lower-energy ones certainly were missed. During each deployment of this sensor, sooner or later it became fouled to the point where it had to be retrieved and cleaned off. The fouling material was almost exclusively macroplankton and was usually either the exoskeleton of copopods or gelatinous material from salpae.

The NBIS planar cell has been mounted with the leading edge canted to the direction of tow, but it also 
suffers the same fouling problem. In this case, the material drapes over the leading edge. A test of this sensor involving a number of deployments resulted in occurrences of fouling about the same as those quoted above for the square cell. The APL/UW sensor, also tested by the authors, performed much better. A number of deployments of this sensor suffered no fouling, and summary percentages are $8 \%$ unusable data and no data of questionable value. The majority of the $8 \%$ was accumulated by one sensor which was fouled for a complete deployment cycle by gelatinous material.

The APL/JHU sensor was designed specifically to avoid this fouling problem and has achieved this objective completely. Farruggia and Fraser (1984) report that it has not suffered a single incident of fouling in more than $100 \mathrm{~h}$ of towing, and our review of some data from this type of sensor confirms this. The UCSD sensor is of a design which would be expected to cause difficulty in this aspect of performance, but the authors report no incidence of fouling. That sensor was deployed only a very limited number of times and suffered a very high noise level, so the report is considered of questionable validity, and we rate this aspect of its performance as unknown.

In Table 1 , this performance is rated only by the qualitative remarks excellent, very good, poor, and unknown because the tests were not sufficiently similar for a quantitative comparison. However, the percentages of fouled data quoted above have a large enough range to permit a reasonable demarcation in their levels of performance of the sensors.

\section{d. Calibration}

Sensor performance with respect to calibration also has to be rated qualitatively since tests have not been identical in every case. The NBIS square cell performs very well, with rms errors of order $0.0003 \mathrm{~S} \mathrm{~m}^{-1}$ reported by Millard (1982), as long as it is not fouled. When fouled, the offset can be as much as $1 \mathrm{~S} \mathrm{~m}^{-1}$ and the calibration can only be restored by cleaning the elec trodes. The typical problem is gelatinous material, which can usually be removed simply by flushing with a stream of clear water. The NBIS planar cell is of fourconductor design and should perform reasonably well in this respect, but it also fouled so often that calibration offsets were a major problem until flushed. The APL/ UW sensor is a two-electrode design and has suffered offsets of up to $0.2 \mathrm{~S} \mathrm{~m}^{-1}$ over 30 days of intermittent deployments at sea. Again, though, the large offsets most likely are due to cases in which the sensor has been fouled. The APL/JHU sensor, as noted previously, is unlikely to foul, and typical offsets during one month at sea are reported by Farruggia and Fraser (1984) to be $0.01 \mathrm{~S} \mathrm{~m}^{-1}$ or less. Finally, the UCSD sensor has a reported drift rate of $0.013 \mathrm{~S} \mathrm{~m}^{-1} / \mathrm{h}$, which is surprisingly high for a four-conductor cell; its cause is unknown. This drift rate was significantly higher than the reported drift rate in a controlled temperature bath, perhaps due to the calibration technique, which depended upon a constant salinity assumption, or it could have been due to impacts of biological material. In either case, it was not high enough to be a problem.

Because of the very different calibration techniques and tests which were used, too much emphasis should not be placed on the specific quantitative values mentioned above. We prefer to rate the cells more qualitatively as excellent, good, or fair, as shown in Table 1.

\section{Conclusions}

The five sensors which are compared here exhibit clear trade-offs in their design objectives. The best spatial resolution is enjoyed by the UCSD cell in principle, but this has not been confirmed in the field, and the sensor exhibited a fair amount of drift and a very high noise level. The drift is not a problem on a short deployment and probably would not cause problems with extended use. It is not certain whether the problem is due to the cell or the electronics. The noise level is a real issue for microstructure applications, but it very likely is due to electronics instead of being inherent in the cell design. The spatial resolution is poorer, in turn, for the needle cell, the NBIS planar cell, the NBIS square cell, and the APL/JHU cell. The latter two are considerably worse than the others.

The noise level of the sensors very likely is due more to the electronics but, for the record, the APL/JHU system is best, followed in turn by the APL/UW needle system, the NBIS systems, and the UCSD system. In terms of fouling immunity, the APL/JHU sensor clearly is the leader, followed closely by the APL/UW needle cell. The NBIS cells are very poor in this regard and the UCSD cell has unknown performance. Finally, the calibration stability is best for the NBIS square cell, followed by the APL/JHU planar cell. The UCSD, NBIS planar and APL/UW cells all drift considerably more, although some of these cells have been deployed intermittently for as long as 30 days, and the total drift was not a problem. It appears that the major cause of calibration drift is impact and subsequent fouling due to material in the water, so that the APL/JHU sensor has an inherent advantage and is the sensor of choice in this respect.

In conclusion, the APL/JHU, APL/UW, and UCSD cells all have specific strengths. The UCSD cell provides the best spatial resolution, at least in principle, and better electronics would be expected to bring its performance up to an acceptable level. The APL/JHU sensor exhibits very low noise level, excellent immunity to fouling and good calibration stability, but its spatial response is quite poor for a microstructure sensor. Finally, the APL/UW sensor provides a reasonable compromise for a microstructure sensor in that the im- 
portant goals of good spatial response, low noise level, and good immunity to fouling are met. The results of this comparison of sensor performance provide the future user of such sensors a considerable base of experience on which to make decisions on an optimum choice for a particular application.

Acknowledgments. Early tests of the APL/UW and NBIS sensors were carried out by B. S. Okawa and T. B. Meagher, and the former affiliation of B. S. Okawa with one of the authors (J. P. Dugan) while at the Naval Research Laboratory naturally led to this current work. His diligence to detail is much appreciated. Also, A. B. Fraser of APL/JHU provided help and unpublished data for obtaining the physical and electrical characteristics of the APL/JHU sensor.

\section{REFERENCES}

Caldwell, D. R., T. M. Dillon, J. M. Brubaker, P. A. Newberger and C. A. Paulson, 1980: The scaling of vertical temperature gradient data. J. Geophys. Res., 85, 1917-1924.

Fabula, A. G., 1968: The dynamic response of towed thermistors. $J$. Fluid Mech., 34, 449-464.

Farruggia, G. J., and A. B. Fraser, 1984: Miniature towed oceanographic conductivity apparatus. Proc. IEEE Conf. Oceans, 10101014.

Garrett, C., 1979: Mixing in the ocean interior. Dyn. Atmos. Oceans, $3,239-265$.

Gibson, C. H., 1981: Buoyancy effects in turbulent mixing: Sampling turbulence in the stratified ocean. Amer. Inst. Aeronaut. Astronaut. J., 19, 1394-1400.

$\longrightarrow$, and W. H. Schwarz, 1963: Conductivity fluctuations in a turbulent flow field. J. Fluid Mech., 16, 357-364.

structure. Air-Sea Interactions: Instruments and Methods, F. Dobson, L. Hasse and R. Davis, Eds., Plenum, 349-368.

Gregg, M. C., and M. G. Briscoe, 1979: Internal waves, finestructure, microstructure and mixing in the ocean. Rev. Geophys. Space Phys., 17(7), 1524-1548.

- and A. Pederson, 1980: High resolution salinity measurement techniques. Air-Sea Interaction: Instruments and Methods, F. Dobson, L. Hasse and R. Davis, Eds., Plenum, 319-348.
- J. C. Schedvin, W. C. Hess and T. B. Meagher, 1982: Dynamic response calibration of the Neil Brown Conductivity Cell. $J$. Phys. Oceanogr., 12, 720-742.

Head, M. J., 1983: The use of miniature four-electrode conductivity probes for high resolution measurement of turbulent density or temperature variations in salt-stratified water flows. Ph.D. dissertation, University of California at San Diego, $211 \mathrm{pp}$.

Lueck, R. G., O. Hertzman and T. R. Osborn, 1977: The spectral response of thermistors. Deep-Sea Res., 24, 951-970.

Magnell, G., 1976: Salt fingers observed in the Mediterranean outflow region $\left(34^{\circ} \mathrm{N}, 11^{\circ} \mathrm{W}\right)$ using a towed sensor. J. Phys. Oceanogr., $6,511-523$.

Marmorino, G. O., J. P. Dugan and T. G. Evans, 1986: Horizontal variability in the vicinity of a Sargasso Sea front. J. Phys. Oceanogr., 16, 967-980.

Meagher, T. B., A. M. Pederson and M. C. Gregg, 1982: A low-noise conductivity microstructure instrument. Proc. IEEE-MTS Conf. Oceans, Washington, DC, 283-290.

Millard, R. C., Jr., 1982: CTD calibration and data processing techniques at WHOI using the 1978 practical salinity scale. MTS Int. STD Conf. and Workshop, La Jolla.

Nasmyth, P. W., 1980: Towed vehicles and submersibles. Air-Sea Interactions: Instruments and Methods, F. Dobson, L. Hasse and R. Davis, Eds., Plenum, 739-765.

Okawa, B. S., and J. P. Dugan, 1984: Contamination of conductivity measurements by waterborne particles. Ocean Eng., 11, 265279.

Pritchard, D. W., 1960: The application of existing oceanographic knowledge to the problem of radioactive waste disposal in the sea. Disposal of Radioactive Wastes, Vol. 2, Proc. Sci. Conf. on Disposal of Radioactive Wastes; Int. Atomic Energy Association, Vienna, p. 229.

Tennekes, H., and J. L. Lumley, 1974: A First Course in Turbulence. The MIT Press, 248-286.

Topham, D. R., and R. G. Perkin, 1984: On the transient behavior of conductivity sensors. J. Atmos. Oceanic Technol., 1(3), 201219.

Washburn, L., and C. H. Gibson, 1982: Measurements of oceanic temperature microstructure using a small conductivity sensor. J. Geophys. Res., 87, 4230-4240.

$\longrightarrow$, and - , 1984: Horizontal variability of temperaturè microstructure at the base of a mixed layer during MILE. J. Geophys. Res., 89, 3507-3522.

- , and T. K. Deaton, 1986: A simple system for mapping conductivity microstructure. J. Atmos. Oceanic Technol., 3(3), 345355. 